\title{
Analysis on the stress of the bone surrounding mini-implant with different diameters and lengths under torque
}

\author{
Yingjuan Lu, Shaohai Chang*, Jiantao Ye, Yushan Ye and Yansong Yu \\ Department of Stomatology, Sun Yat-sen Memorial Hospital, Sun Yat-sen University, Guangzhou \\ 510120, Guangdong Province, China
}

\begin{abstract}
The purpose of this study is to compare the stress of the bone around the mini-implant under the two kinds of force: the composite force which contains torque and traditional single force. There were 96 finite element models formed by the combination of mini-implant and bone, with diameters of $1.2 \mathrm{~mm}, 1.6 \mathrm{~mm}, 2.0 \mathrm{~mm}$ and corresponding length being $6 \mathrm{~mm}, 8$ $\mathrm{mm}, 10 \mathrm{~mm}, 12 \mathrm{~mm}$, respectively. Each size corresponded to 8 models. Group SF (each size $\mathrm{n}=4$ ) was loaded with $200 \mathrm{~g}$ single force, while Group CF (each size $\mathrm{n}=4$ ) was loaded with composite force which contained $6 \mathrm{~N}$ mm torque and $200 \mathrm{~g}$ single force. The maximum equivalent stress (Max EQS) of the bone surrounding mini implant with different loading directions was calculated, and the relationship of force direction, diameter and length was also evaluated. The Max EQS of Group CF was higher than that of Group SF. The effect of force direction on the stress was related to the diameter of mini implant, but had nothing to do with its length. The Max EQS of the cortical bone around mini implant in Group CF was higher $(\mathrm{P}<0.05)$ than that in Group SF. In contrast, there was no significant difference $(\mathrm{P}>0.05)$ between Group SF and Group $\mathrm{CF}$ in terms of bone stress when the diameter of mini implant was $1.6 \mathrm{~mm}$ or $2.0 \mathrm{~mm}$. In our study, it is demonstrated that the diameter of mini-implant is better to be larger than $1.2 \mathrm{~mm}$ when a mini-implant is used in a torque control of tooth. The impact of this feature in the clinical setting needs to be verified.
\end{abstract}

Keywords: Mini-implant, torque, diameter, length

\section{Introduction}

As a new tool to strengthen the anchorage, the mini implant has become increasingly common in orthodontic treatment. Scholars have always been working hard on how to improve the success rate of the mini-implant which is greatly influenced by the stress of the bone around the mini-implant. Research at the present stage is much concerned with the control of teeth through the mini-implant in single direction, and considers that the appearance of mini-implant can affect the stress in terms of diameter, length and so on.

The upright of tilted molar has always been a difficult problem in orthodontic treatment, because the center of resistance of the tooth is basically consistent with the geometric center of the tooth root, the

\footnotetext{
* Address for correspondence: Shaohai Chang, Department of Stomatology, Sun Yat-sen Memorial Hospital, Sun Yat-sen University, Guangzhou 510120, Guangdong Province, China. Tel.: +86 020-81332099/+86 020-34071070; Fax: +86 020-81332505; E-mail addresses: changhaoh@gmail.com.
} 
center of resistance of multi-root tooth lies in the root furcation to the direction of root tip about $1-2 \mathrm{~mm}$ and its location changes with the length of the tooth root. When it imposes single force on the molar crown, only tipping movement occurs in the tooth because the center of resistance is beneath the point of application, and it is very hard to use traditional methods to make those tilted molars upright in both mesiodistal and buccolingual directions.

In recent years, scholars have found that imposing the composite force containing the torque on the mini-implant will result in the motion of the tooth in a three dimensional direction [1,2]. D.M. Guo, et al. applied this method to make the tilted molar upright and found that it has good dimensional control on the mesiobuccal tilted molar. Due to the mini-implant, the moved tooth is a partial correction system which helps avoid the adverse effects of the other teeth as anchorage [3]. But under this force, there are no related reports about the stress of the bone around the mini-implant. This study compares the stress of the bone around the mini-implant under the two kinds of force: the composite force which contains torque and traditional single force.

\section{Material and methods}

The maxilla Computed Tomography (CT) data of volunteers were saved in DICOM format and imported into Mimics 10.0 software (Macrovision Corporation, USA), which set an appropriate threshold, extracted soft tissue and hard tissue, and proceeded the three-dimensional reconstruction of the hard and soft tissues respectively. Interference of the neck structure and head was eliminated when performing the CT scan and the interference of the metal artifacts was removed at the same time. The maxillary model was then generated, and Geomagic Studio 8.0 (Raindrop Geomagic, USA) was used to refine the model. Finally, the model was exported in the stp format for editing.

The mini-implant was based on Tomas mini-implant (Dentaurum, Germany) as it is the most commonly used mini-implant size in clinical setting. Unigraphics NX 6.0 (Siemens Product Lifecycle Management Software, USA) was used to build the three-dimensional model of micro-implant. The three dimensional solid model of mini-implant with diameters of $1.2 \mathrm{~mm}, 1.6 \mathrm{~mm}, 2.0 \mathrm{~mm}$, and corresponding length of $6 \mathrm{~mm}, 8 \mathrm{~mm}, 10 \mathrm{~mm}, 12 \mathrm{~mm}$ were established.

The mini-implant was inserted into the buccal alveolar bone of maxillary second molar at $10 \mathrm{~mm}$ from mesial alveolar ridge and closed at $6 \mathrm{~mm}$ from the alveolar crest [3]. The mini-implant placement orientation was 90 degrees, and 96 maxilla models which contained the micro-implant were built, with each size of micro-implant corresponding to 8 models. The thickness of mandibular cortical bone ranged from 1.5-2.0 $\mathrm{mm}$. Suppose that there was a mechanical bond between the mini-implant and jaw which means that these two were frictional contact, the friction coefficient was $0.3[4,5]$.

The integrated plug in board in ANSYS Workbench 13.0 (SAS, USA) and Unigraphics were used to import the model into ANSYS Workbench, and the diameter and length parameters which were set simultaneously should be also passed to the analysis software. Tetrahedral mesh was used to divide the cortical bone, cancellous bone and screws and to improve the quality of the grid. The model nodes ranged from 168770-223077, and unit number ranged from 107446-143600.

The force situation of mini-implant in clinical setting was simulated, loaded near middle single force $200 \mathrm{~g}$ and composite force that is $200 \mathrm{~g}$ single force plus $6 \mathrm{~N} \mathrm{~mm}$ torque. Four of each size were randomly selected to load with single force, the other 4 were composite force loaded.

This study was to simulate III bone in the jaw classification of Lekholm and Zarb. All the materials were assumed to be homogeneous, isotropic linear elastic material. The material parameters were illustrated in Table 1. 
Table 1

Material properties of bones and mini implant [6]

\begin{tabular}{lll}
\hline Material & Young's moduls (MPa) & Poisson's ratio \\
\hline Cortical bone & 13,700 & 0.33 \\
\hline Cancellous bone & 1,600 & 0.3 \\
\hline Mini implant & 110,000 & 0.35 \\
\hline
\end{tabular}

Table 2

Independent effect of force on Max EQS in cortical bone

\begin{tabular}{lllll}
\hline Diameter & Force & $\bar{X} \pm S$ & $t$ & $P$ \\
\hline 1.2 & SF & $23.50 \pm 2.76$ & -2.89 & 0.007 \\
& CF & $26.89 \pm 3.79$ & & \\
1.6 & SF & $12.26 \pm 2.60$ & 0.149 & 0.882 \\
& CF & $12.13 \pm 2.31$ & & \\
2.0 & SF & $6.92 \pm 1.09$ & -1.762 & 0.088 \\
& CF & $7.69 \pm 1.38$ & & \\
\hline
\end{tabular}

The Maximum Equivalent Stress of cortical bone and cancellous bone of mini-implant in different sizes under the two groups of forces was measured and statistic analysis was made by putting the results into the SPSS 17.0.

\section{Results}

Due to the three factors designed in the experiment: direction, diameter, and length, factorial analysis on those factors should be made first. The MANOVA analysis of between-group effects are used to show the interaction. It was found that there wasn't secondary interactive effect among stress direction, diameter and length, but the primary interactive effect existed. If there was an interactive effect, the independent effect of experimental factors needed to be analyzed. The t test was used to analyze the difference between two force groups (Table 2). The Max EQS was significantly different in the two force type groups with an implant diameter of $1.2 \mathrm{~mm}(\mathrm{P}=0.007)$.

\section{Discussion}

The contact between the micro-implant and the jaw was set as the fixed contact in the past [7]. But in clinical work, especially in the condition of the instant load, there is only a mechanical bond between micro-implant and jaw and there exists mobility in it. This study set these two contact relations as the frictional contact, and the friction coefficient was set as $0.3[4,5]$, which is more in line with the actual clinical situation.

The steady mini-implant can bear at most $300 \mathrm{~g}$ orthodontic single force, and the force value which is loaded instantly should not exceed $200 \mathrm{~g}[8,9]$. As for the torque control of the posterior teeth, Chan and Hohmann thought the torque more than $6 \mathrm{Nmm}$ would cause root resorption $[10,11]$. This study mainly focused on the stress distribution in the physiological state. Single force and torque were loaded for the center of the structure of the head of mini-implant by the loading parts, with load size being $200 \mathrm{~g}$ and $6 \mathrm{~N} \mathrm{~mm}$ respectively, and the load imposed in this experiment was quite similar to the situation in the clinical work. 
As previous studies on the dimensional finite element were generally a descriptive study of a single sample, namely the establishment of a model to describe the value of each index, its results had a certain limitation. This study established a 96-group model, a professional statistical analysis, experimental repeatability, which increased the persuasiveness of the results. A lower Max EQS indicates less stress on the surrounding bone, which implies less damage to the bone and higher success rate of the micro-implant $[12,13]$. The result shows that there is an interactive effect between the force direction and the diameter, so the effects of force direction on the same diameter need to be analyzed separately.

When diameter was $1.2 \mathrm{~mm}$, the difference between the two groups of forces was statistically significant. But when diameter was $1.6 \mathrm{~mm}$ or $2.0 \mathrm{~mm}$, the difference was not statistically significant. To determine whether the primary stability of mini-implant is affected, the variations of the above two observations should be considered comprehensively. So the difference between the jaw stress values under the single force groups and the composite forces groups existed. In addition, under the loading of composite forces, the stress value of the bone is higher than that in the single force. Therefore, the previous results indicated that composite forces have unfavorable effects on the stress of bone surrounding mini- implant.

There wasn't interactive effect between force direction and the length, which means that the length does not change the effects that the force direction works on the stress. However, there existed interactive effect between force direction and the diameter. Table 2 showed that when diameters were different, the amplitude response lines of cortical bone Max EQS towards force directions were different. When the diameter was $1.2 \mathrm{~mm}$, the effect of force direction on the observation index was more obvious than that with diameters of $1.6 \mathrm{~mm}$ or $2.0 \mathrm{~mm}$. It was known that the adverse effects of the composite force on the jaw stress mainly occur around the mini-implant of small diameter. This may relates to the fact that the force arm increases with the increasing diameter, under a fixed torque, which leads to the decrease of the stress of bone.

\section{Conclusion}

Composite forces have unfavorable effects on the stress of bone surrounding the mini implant. In the present study, the length of mini-implant will not change the force direction's effects on the stress of bone surrounding the mini-implant. But there are some unfavorable effects of the composite forces on the stress of bone surrounding the mini-plant with a smaller diameter. When the diameter of the mini-plant is larger than $1.2 \mathrm{~mm}$, the experiment of putting composite forces with torque on the mini-implant is more likely to achieve success. These results of the study are merely based on a computer model-experiment and need to be further verified in the clinical setting.

\section{Acknowledgments}

This study was granted from Sun Yat-sen Memorial Hospital, Guangdong Province, China.

\section{References}

[1] H. De Clerck, M. Cornelis and H. Timmerman, Dental tours de forces 4, the use of a bone anchor for holding upright a tipped molar in the lower jaw, Nedrlands tijdschrift voor tandheelkunde 111 (2004), 10-13. 
[2] K.R. Chung, S.H. Kim, M.P. Chaffee, et al., Molar distalization with a partially integrated mini-implant to correct unilateral Class II, American Journal of Orthodontics and Dentofacial Orthopedics 138 (2010), 810-819.

[3] D.M. Guo, S.H. Chang, L.L. Hu, et al., Biomechanics of upper molar uprighting with Tomas micro-implant-a finite element study, Chinese Journal of Orthodontics 19 (2012), 86-91.

[4] J. Ao, T. Li, Y. Liu, et al., Optimal design of thread height and width on an immediately loaded cylinder implant: A finite element analysis, Computer in Biology and Medicine 40 (2010), 681-686.

[5] N. Woodall, S.C. Tadepalli, F. Qian, et al., Effect of miniscrew angulation on anchorage resistance, American Journal of Orthodontics and Dentofacial Orthopedics 139 (2011), 147-152.

[6] M.I. Jasmine, A.A. Yezdani, F. Tajir, et al., Analysis of stress in bone and micro implants during en -masse retraction of maxillary and mandibular anterior teeth with different insertion angulations: A 3-dimensional finite element analysis study, American Journal of Orthodontics and Dentofacial Orthopedics 141 (2012), 71-80.

[7] M. Motoyoshi, M. Inaba, S. Ono, et al., The effect of cortical bone thickness on the stability of orthodontic miniimplants and on the stress distribution in surrounding bone, International Journal of Oral and Maxillofacial Surgery 38 (2009), 13-18.

[8] S.J. Cheng, I.Y. Tseng, J.J. Lee, et al., A prospective study of the risk factors associated with failure of mini-implants used for orthodontic anchorage, The International Journal of Oral and Maxillofacial Implants 19 (2004), 100-106.

[9] J.S. Garfinkle and C.S. Beeman, Evaluation of orthodontic mini-implant anchorage in premolar extraction therapy in adolescents, American Journal of Orthodontics and Dentofacial Orthopedics 133 (2008), 642-653.

[10] E. Chan and M.A. Darendeliler, Physical properties of root cementum: Part 5, Volumetric analysis of root resorption craters after application of light and heavy orthodontic forces, American Journal of Orthodontics and Dentofacial Orthopedics 127 (2005), 186-195.

[11] A. Hohmann, U. Wolfram, M. Geiger, et al., Periodontal ligament hydrostatic pressure with areas of root resorption after application of a continuous torque moment, The Angle orthodontist 77 (2007), 653-659.

[12] S. Singh, S. Mogra, V.S. Shetty, et al., Three-dimensional finite element analysis of strength, stability, and stress distribution in orthodontic anchorage: a conical, self-drilling miniscrew system, American Journal of Orthodontics and Dentofacial Orthopedics 141 (2012), 327-336.

[13] J. Liu, S. Pan, J. Dong, et al., Influence of implant number on the biomechanical behavior of mandibular implant retained/supported overdentures: A three-dimensional finite element analysis, Journal of Dentistry 3 (2013), 241-249. 